\title{
Serial overshadowing of taste aversion learning by stimuli preceding the target taste
}

\author{
Dorothy W. S. Kwok • Evan J. Livesey • \\ Robert A. Boakes
}

Published online: 11 January 2012

(C) Psychonomic Society, Inc. 2012

\begin{abstract}
Three experiments tested whether events taking place before a rat has access to a target taste, sucrose, can proactively interfere with the acquisition of a sucrose aversion when sucrose is followed by a lithium chloride injection. Using a serial overshadowing procedure with various delays before lithium injection, proactive interference by a taste (Experiments 1 and 3) and by a novel context (Experiment 2) was found following two conditioning sessions, but not after a single conditioning session. Conversely, overshadowing by a taste given after the target was detectable after a single conditioning trial (Experiment 3) and, thus, indicated that retroactive interference involves a process different from that producing proactive interference. A simulation confirmed that the results are consistent with a modified Rescorla and Wagner (1972) interpretation of Revusky's (1971) concurrent interference theory of delay learning.
\end{abstract}

Keywords Conditioned taste aversion · Long-delay learning - Serial overshadowing · Proactive interference .

Retroactive interference $\cdot$ Context conditioning

Optimal learning tends to be obtained with very short interstimulus intervals (Kimble, 1961; Mackintosh, 1974). A classic example is the finding that delaying a food reward for a correct turn by just half a second severely interfered

\footnotetext{
D. W. S. Kwok • E. J. Livesey

University of Sydney,

Sydney, Australia

R. A. Boakes $(\bowtie)$

School of Psychology (A18), University of Sydney,

Sydney, NSW 2006, Australia

e-mail: bob.boakes@sydney.edu.au
}

with maze learning in rats (Grice, 1948). A more recent example from human learning research is the finding that action-outcome delays of more than $2 \mathrm{~s}$ greatly interfered with performance of a causal learning task (Shanks, Pearson, \& Dickinson, 1989). Following Pavlov (1927), these effects have been most commonly attributed to the decay over time of a stimulus trace. The major exception to the generalization that learning to associate two events requires that they are separated by no more than a minute or so comes from research on conditioned taste aversions. In the first experiment to demonstrate learning despite a delay of some hours between a taste and a nausea-inducing event, rats were given a single pairing between saccharin and a sublethal dose of $\mathrm{x}$-radiation given up to $12 \mathrm{~h}$ later and, subsequently, displayed aversion to the saccharin (Garcia, Kimeldorf, \& Koelling, 1955). Since that study, a large number of experiments have confirmed that taste aversion learning can be obtained despite delays of many hours (Freeman \& Riley, 2009).

The first, and apparently only, attempt to explain such long-delay learning within a general framework is provided by Revusky's (1971) concurrent interference theory. This proposes that long-delay learning between two events is possible in any domain to the extent that other events do not interfere with the reference association; thus, the common difficulty in obtaining conditioning effects as a delay is increased arises from increased opportunity for interference by other events, rather than resulting from the rapid decay of some memory trace. Because nausea is selectively associated most strongly with tastes and very weakly with audio-visual events, as first reported by Garcia and Koelling (1966), in a long-delay taste aversion experiment, there are normally few events intervening between the target taste and the sicknessinducing event that can interfere at all effectively with acquisition of an aversion to the taste. However, as Revusky clearly 
demonstrated, giving a rat an additional taste during this interval can strongly interfere with - and under some conditions, completely prevent-acquisition of an aversion to the target taste.

Revusky (1971) described the kind of interference described so far as retroactive interference, because the potentially interfering event occurs in the interval between the target conditioned stimulus (CS) and the unconditioned stimulus (US). Traditional research on interference effects in human memory has distinguished between the impact of retroactive interference on forgetting (McGeoch \& Irion, 1952) and the sometimes even stronger impact of proactive interference (Underwood, 1957). In the present context, this refers to the influence of events occurring prior to the target CS. Although Revusky proposed that events occurring within the interval between two events (which he labeled "E-pre" and "E-post," but here are referred to as the target CS and the US) produce greater interference than do events occurring outside the CS-US interval, the latter, he claimed, may also produce interference.

Revusky (1971) included brief reports of two experiments that provided some evidence for proactive interference. On each of three conditioning days, the rats were first given saccharin or water and, 15 min later, access to either coffee or vinegar solution as the target taste; after a further $60 \mathrm{~min}$, they received an injection of lithium chloride $(\mathrm{LiCl})$. A conditioned aversion was tested by giving repeated two-bottle tests between the target taste (e.g., coffee) and the nonpaired taste (e.g., vinegar). The procedures were complex, including preexposure to both coffee and vinegar, and the evidence for proactive interference from these two experiments is far less convincing (Revusky, 1971, pp. 192-197) than his evidence for retroactive interference. It is worth noting that there have been several subsequent demonstrations of retroactive interference in taste aversion learning, including, as is discussed below, interference from contextual stimuli. However, only one published study since Revusky has examined proactive interference in taste aversion learning.

Bond (1983) gave rats two tastes in succession - vinegar, then saline - followed by lithium injection $2 \mathrm{~h}$ later, in a one-trial serial overshadowing design. Subsequent tests for aversions to both vinegar and saline indicated reciprocal overshadowing, in that - to use Revusky's (1971) terminology - both retroactive and proactive interference were obtained. Proactive interference was obtained when vinegar immediately preceded saline in the conditioning session (Experiment 1), but when a 10-min delay was introduced, there was no interference by vinegar with the acquisition of an aversion to saline (Bond, 1983, Experiment 2). This led Bond to conclude that the proactive interference detected in his experiments was due to generalization decrement, in that the taste of saline immediately after a rat had consumed vinegar, as in the conditioning session, was probably different from the taste of saline in a test session that did not contain any preceding vinegar.

We have recently found that events other than tastes that take place before a rat has access to a taste can interfere with a rat's acquisition of an aversion to that taste. Using a context blocking design, rats were initially given context discrimination training whereby placement in a novel context (target) was followed by lithium injection, while placement in a second, distinctive context (safe) was followed by saline injection. In the subsequent one-trial taste conditioning session, rats were first placed either in their target (blocking group), a safe, or a neutral context that had never been paired with an injection and were then given access to sucrose, which was followed by an injection of lithium 30 min later. A subsequent test revealed a weaker sucrose aversion in the blocking group than in the two control groups (Kwok \& Boakes, in press). It seems very unlikely that placement in a distinctive context would affect the taste of the solution subsequently consumed in a second context. Therefore, this result encouraged the search for proactive interference based on overshadowing under conditions where it is unlikely that interference would result simply from generalization decrement.

The first aim of the present study was to examine whether more robust evidence for proactive interference based on overshadowing can be obtained than that reported by Revusky (1971) under conditions that effectively rule out a generalization decrement account (cf. Bond, 1983). The second aim was to test whether any such interference might increase with the delay between the target taste and lithium injection. Evidence for such a relationship comes from both human and animal research. For example, many human studies have found that, when people learn list $\mathrm{A}$ at time $t_{1}$ followed by learning a similar list, $\mathrm{B}$, at time $t_{2}$ and then are asked to recall list $\mathrm{B}$ at time $t_{3}$, forgetting of $\mathrm{B}$ due to intrusions from A (i.e., proactive interference) increases as the interval between $t_{2}$ and $t_{3}$ is increased (Baddeley, 1990; Loftus, 1980; Postman, 1975). An example of the same relationship in animal memory has been provided by Gleitman and Jung (1963). They trained rats to respond first on one of two levers and then on the other lever. Retention intervals of 1 or 44 days following training were compared. For two control groups that been trained on a single lever, no forgetting was found regardless of retention interval. In contrast, rats that had been trained on both responses exhibited some forgetting of the most recently trained response - that is, they responded on the lever that they had initially been trained to choose - with those that were tested after the 44-day interval showing the most forgetting.

In the light of such research on proactive interference in other paradigms, we included a long and a short delay in Experiments 1 and 2, with the prediction that proactive interference, reflected in relatively weaker conditioning of 
the target taste, would be greater with the longer delay. The target taste in all three experiments was provided by the same sucrose solution.

\section{Experiment 1: Overshadowing of sucrose aversion learning by a pre-CS taste}

This experiment used a $2 \times 2$ factorial design in which one factor was whether or not access to the target taste, sucrose, was preceded by the potentially overshadowing taste of saline on conditioning days. The second factor was whether access to sucrose was followed by a lithium injection either 15 or 90 min later. Because we did not wish to confound possible differences in overshadowing across the two delay intervals with differences in the strength of the aversion shown by the two control groups, the lithium dose was made stronger for the two 90-min groups.

An important way in which the present procedure differed from that used by Bond (1983) was that two conditioning trials were given. This was because a previous experiment had failed to find any indication of overshadowing following a one-trial conditioning procedure (Kwok, 2010), thus confirming Bond's failure to find overshadowing by a pre-CS taste when this preceded the target taste by $10 \mathrm{~min}$.

\section{Method}

Subjects Thirty-two male albino Wistar rats, obtained from ARC Perth, were approximately 120 days old at the start of the experiment, when their mean weight was $563 \mathrm{~g}$ (range, 466$663 \mathrm{~g}$ ). The previous experiment in which they had served did not involve exposure to any tastes, to any nausea-inducing substances, or to strongly aversive stimuli. The rats were housed in squads of four in large plastic tubs, $37 \times 25 \times$ $53 \mathrm{~cm}$ high. Standard laboratory rodent food was continuously available throughout the experiment. Lighting operated on a normal 12:12-h light:dark cycle, with lights on at $0800 \mathrm{~h}$. Experimental sessions started about $2 \mathrm{~h}$ into the light period.

Immediately prior to the start of the experiment, an initial phase of water deprivation was conducted whereby, over a 4day period, access to water in the colony room was progressively reduced from $4 \mathrm{~h}$ to $30 \mathrm{~min}$. Thereafter, supplementary access to water was maintained at $30 \mathrm{~min} /$ day, delivered upon returning to the colony room following a daily session.

Apparatus and solutions A separate laboratory contained eight transparent acrylic cages, $33 \times 21 \times 19 \mathrm{~cm}$ high, referred to here as the neutral drinking chambers. Flooring was commercial cat litter. Plastic drinking bottles of 300-ml capacity with a rubber stopper and stainless steel ballbearing spout could be attached to the acrylic cages using a metal bracket. The spout entered the chamber at an angle of $45^{\circ}$ through the steel wire roof. Fluid intakes were measured by weighing bottles to the nearest $0.1 \mathrm{~g}$ before and after each session. Drinking solutions were $8 \%$ sucrose and $0.5 \%$ saline, both in tap water.

Procedure Experimental sessions were conducted 7 days per week. During water pretraining on days $1-3$, all rats were given water in the drinking chambers, with access progressively reduced from 20 to $10 \mathrm{~min}$. They were matched for water intake on day 3 and counterbalanced for prior experience when allocated to the four experimental groups (each $n=8$ ): Saline- $15 \mathrm{~min}$, Control-15 min, Saline-90 min, and Control-90 min. On day 4, the control groups were preexposed to the saline solution in a 10-min session in the drinking chambers, in order to reduce any neophobia when these rats were given saline in the test stage. The saline groups were given 10-min access to water on day 4.

Conditioning took place on days 5 and 7. On each of these days, the saline groups were given saline for $5 \mathrm{~min}$ in the drinking chambers, returned to their home cages for $10 \mathrm{~min}$, and then placed back in the drinking chambers, where they had access to sucrose for $5 \mathrm{~min}$. The Saline15-min group was given an intraperitoneal (ip) injection of $0.15 \mathrm{M} \mathrm{LiCl}$ at $4 \mathrm{ml} / \mathrm{kg} 15 \mathrm{~min}$ later, while the Saline-90min group was given an injection of $0.15 \mathrm{M} \mathrm{LiCl}$ at $9 \mathrm{ml} / \mathrm{kg}$ 90 min later. The lithium doses were chosen on the basis of a prior study and were intended to produce the same moderate level of taste aversion in the Control-15-min and Control90-min groups. Individual doses were based on body weight on the day prior to conditioning. The procedures for the Control-15-min and Control-90-min groups were exactly the same, except that saline was replaced by water. On conditioning trials, animals were returned to their home cages after drinking the sucrose solution and remained there until they were injected. Amounts of sucrose, saline, and water were limited to $10 \mathrm{ml}$ for these two sessions.

On days 6,8 , and 9 , all rats received water in the drinking chambers exactly as during pretraining. On day 10 , rats were given unlimited access to sucrose. This test session lasted $10 \mathrm{~min}$.

Statistical analyses consisted of ANOVAs. Results from all three of the present experiments were considered significant when $p<.05$.

Results and discussion

The four groups did not differ in water intake on the final pretraining day (day 3), $F_{\mathrm{S}}<1$, when the mean (and $S E M$ ) intakes were the following: Saline-15 min, 12.9 (1.3) ml; Control-15 min, 11.5 (1.2) ml; Saline-90 min, 12.4 (0.5) $\mathrm{ml}$; and Control-90 min, $12.1(0.8) \mathrm{ml}$. These intakes were compared with water intakes in the session immediately 
prior to the testing session (day 9) as a check on whether there might be differences in context conditioning across the groups. A $2 \times 2 \times 2$ mixed ANOVA did not detect any main effects of group, delay, or session or any interactions, all $F$ s $<1$. Thus, water intakes effectively did not change from before to after the conditioning treatment, and there were no differences between the groups on this measure. These results suggest that the rats had not acquired any appreciable aversion to the drinking context, and to the extent that there might have been a slight aversion, it did not differ between the groups.

Sucrose intakes in the two conditioning sessions (days 5 and 7) and the test session (day 10) are shown in Fig. 1. It may be seen that there was essentially no difference between the saline and control groups in either conditioning session but that, on day 10 , the saline groups drank more than their control groups. When $2 \times 2$ ANOVAs were applied to intakes during the two conditioning sessions, no effect of group or delay and no interaction were detected, all $F_{\mathrm{S}}<1$; thus, there was no indication that the sucrose aversion produced by a single trial was affected by whether or not the rats had been given saline prior to the sucrose. The same analysis applied to sucrose intakes at test (day 10) revealed a main effect of the group (saline vs. control) factor, $F(1,28)=16.79$, thus confirming that there was a greater reduction of sucrose intakes in the control groups than in the saline groups and, so, demonstrating overshadowing by saline of the sucrose aversion. Neither the delay nor the interaction involving this factor was significant, both $F \mathrm{~s}<1$. Thus, the prediction that interference by saline would be greater for the longer delay was not supported, even though conditioning was as effective over the 90 -min delay as over the 15 -min delay.

In summary, these results demonstrated that overshadowing of taste aversion learning by a pre-CS taste can be detected after two conditioning trials. The contrasting failure

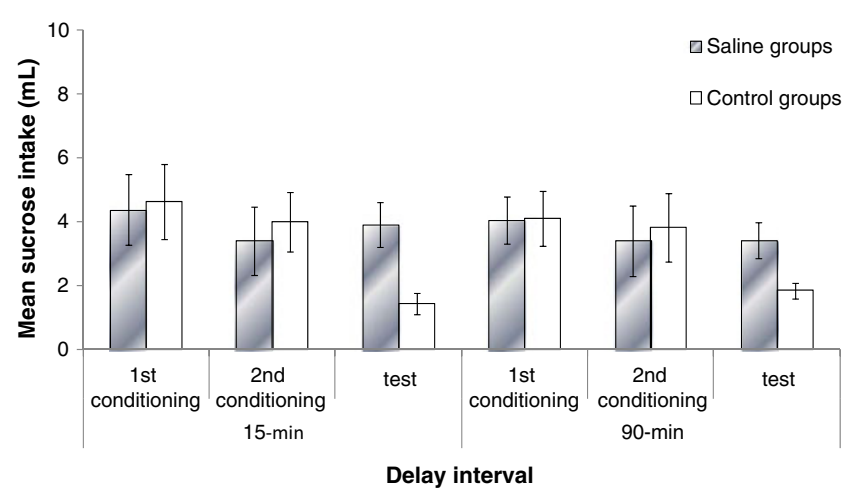

Fig. 1 Experiment 1: Sucrose intakes during the two conditioning sessions and the test session. The two saline groups were given saline prior to sucrose, while the control groups were given water at this time. Lithium injections were given either 15 or 90 min following access to sucrose to find an overshadowing effect after a single trial might indicate only that the conditions for the second conditioning session (day 7)-for example, limiting fluid access to 5 min-were not sensitive enough for detecting such an effect. However, the absence of one-trial overshadowing is consistent with the same result from a previous experiment that included only a single conditioning trial (Kwok, 2010) and also with the results from Experiment 3, reported below. As is discussed in more detail in the General Discussion section, detection of an overshadowing effect only after at least a second conditioning trial is consistent with Rescorla and Wagner's (1972) analysis of overshadowing. Finally, there was no evidence that extending the delay from 15 to 90 min increased overshadowing by saline, even though the differences in lithium doses produced similar degrees of sucrose aversions across the delays.

\section{Experiment 2: Context overshadowing of a conditioned taste aversion}

As was noted earlier, Kwok and Boakes (in press) found that placing rats in a context previously associated with lithium injection, before given them access to a target taste followed by lithium, produced a blocking effect in that aversion to the target taste was reduced, relative to controls. The present experiment tested whether exposure to a novel and distinctive context prior to the target taste would overshadow aversion to the taste. The design was essentially the same as that of Experiment 1, except that on the two conditioning days, rats in the experimental condition were placed in a distinctive context, rather than being given a second taste, before being given sucrose. Lithium injection was given either 15 or 90 min following sucrose, as in Experiment 1.

\section{Method}

Subjects Thirty-two male albino Wistar rats were from the same source as that in Experiment 1 and had a similar prior experimental history. They were 142 days old at the start of the experiment, with a mean weight of $558 \mathrm{~g}$ (range, 432 $660 \mathrm{~g}$ ). General procedures, including housing, food, gradual water deprivation, and subsequent access to water, were the same as in Experiment 1.

Apparatus and solutions The eight drinking chambers used in the previous experiment and made of clear acrylic, with steel mesh tops and cat litter covering the floor, are referred to here as the neutral context. An additional set of eight cages served as the experimental context. These were steel cages with steel rod flooring, $20 \times 20 \times 30 \mathrm{~cm}$ high. No odors were added to enhance the differences between the two sets of cages. Thus, we assume that to discriminate 
between them, rats relied on the cages' different positions in the laboratory or on the differences in visual, tactile, and auditory stimulation that they provided. The target taste was again provided by $8 \%$ sucrose.

Procedure Pretraining with water in the drinking chambers (days 1-5) was exactly as in the previous experiment, except that two additional water sessions were included. These additional sessions and all subsequent drinking sessions lasted $10 \mathrm{~min}$. Rats were allocated to the four groups, matching for water intake on the last day of pretraining and counterbalanced for previous experimental history; the groups were designated Context-15 min, Control-15 min, Context-90 min, and Control-90 min.

In the two conditioning sessions, access to sucrose was followed by lithium injection either 15 or 90 min later (days 6 and 8 ). On both these days, rats in the context groups were placed in the steel cages for $10 \mathrm{~min}$, returned to their home cages for $1 \mathrm{~min}$, and subsequently placed in the drinking chambers, where they had access to sucrose for $5 \mathrm{~min}$. Rats in the control groups were placed in the familiar drinking chambers for $10 \mathrm{~min}$ without access to any fluid, returned to the home cages for $1 \mathrm{~min}$, and then returned to the drinking chambers, where they also had access to sucrose for $5 \mathrm{~min}$. As in Experiment 1, the two groups in the 15-min condition were given an injection of $\mathrm{LiCl}$ at $4 \mathrm{ml} / \mathrm{kg} 15 \mathrm{~min}$ after being given sucrose, while the two groups in the 90-min condition were injected with $\mathrm{LiCl}$ at $9 \mathrm{ml} / \mathrm{kg} 90 \mathrm{~min}$ after sucrose access. On day 7 , rats were given water in the drinking chambers, as during pretraining. The only difference between the first and second conditioning trials (days 6 and 8 ) was that the amount of sucrose available was increased from $10 \mathrm{ml}$ on day 6 to $40 \mathrm{ml}$ on day 8 . This was to prevent any group differences that might emerge following the first conditioning trial from being obscured by a low ceiling on the second conditioning trial. All rats were given water in the drinking cages for two sessions (days 9 and 10) following the second conditioning session.

Rats were tested on day 11, in which they were placed in the drinking chambers and given effectively unlimited access to sucrose for $10 \mathrm{~min}$.

\section{Results and discussion}

As in the previous experiment, water intakes on the last session of pretraining (day 5), on day 7, and on the 2 days following the second conditioning trial were analyzed to test for possible differences between the groups that might indicate differential aversion to the neutral drinking chambers. On the final pretraining day, the mean (and SEM) water intakes for the groups were the following: Context-15 min, 14.7 (1.6) $\mathrm{ml}$; Control-15 min, 15.7 (1.1) ml; Context-90 min, 15.8 (0.8) $\mathrm{ml}$; and Control-90 min, 15.5 (1.6) ml. A $2 \times 2 \times 4$ ANOVA showed no differences between groups and delays or across sessions and no interactions, all $F_{\mathrm{S}}<1$.

The important results are shown in Fig. 2 for sucrose intakes in the first and second conditioning sessions and in the initial sucrose test session (day 11). It may be seen that no sign of overshadowing appeared in the second conditioning session, in that context groups drank the same small amount of sucrose as the control groups. However, in the test session, the Context-15-min group drank more than the Control-15-min group, thus indicating overshadowing by the context. On the other hand, this test did not reveal any such effect in the Context-90-min group.

This description of the results was confirmed by the following analyses. When group $\times$ delay ANOVAs were applied to intakes in the conditioning sessions, no main effects and no interaction were found in either the first conditioning session, largest $F(1,28)=1.90$, or the second, all $F \mathbf{s}<1$. On the other hand, the same analysis applied to intakes in the test showed a main effect of context versus control, $F(1,28)=6.89$, and an interaction between this factor and delay, $F(1,28)=4.86$, but no main effect of delay, $F<1$. Subsequent tests for simple effects showed that the Context-15-min group drank more sucrose than did the Control-15-min group, $F(1,28)=18.46, \mathrm{p}<.01$, but there was no difference between the Context-90-min and Control-90-min groups, $F<1$.

This failure to find overshadowing by a context when a 90-min delay was employed contrasts with the finding in Experiment 1 of overshadowing by a taste at the same interval. As is discussed further in the General Discussion section, we suggest that this difference is because the "trace" of the context in the present experiment decayed more over $90 \mathrm{~min}$ than did the trace of the taste used in Experiment 2.

It was noted that the failure in Experiment 1 to detect overshadowing after a single conditioning session might

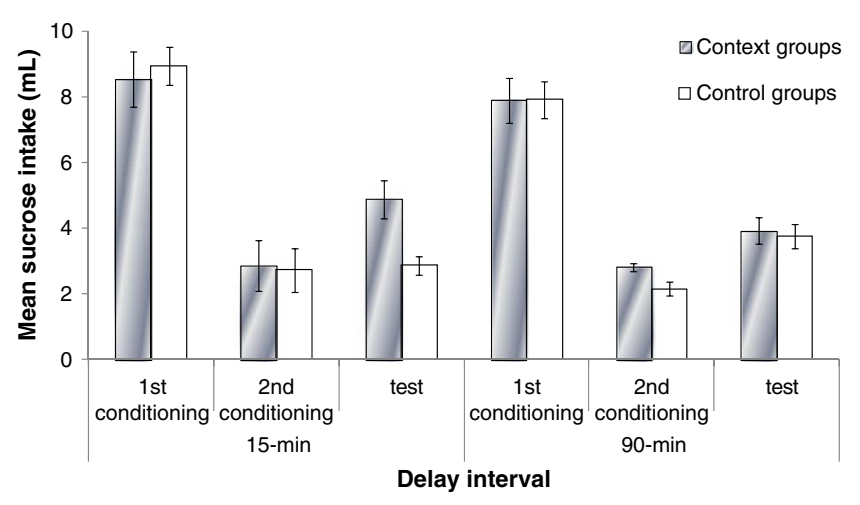

Fig. 2 Experiment 2: Sucrose intakes during the two conditioning sessions and the test session. In both conditioning sessions, the two context groups were placed in a distinctive context before being given sucrose, while the control groups were given only equivalent handling before sucrose. Lithium injections were given either 15 or $90 \mathrm{~min}$ following access to sucrose 
simply reflect insufficient sensitivity of the conditions used in the second conditioning session. A similar point can be made in relation to the two 15-min groups in the present experiment and the absence of any difference between them - that is, no overshadowing - in the second conditioning session. In this case, one might point to the possible role of placement of the Context-15-min group in the steel cages $10 \mathrm{~min}$ before being given access to sucrose in the highly familiar drinking cages. It is possible that the context shift tended to reduce subsequent sucrose intakes by the Context-15-min group to an extent that just counteracted the increase in intakes that would be produced by one-trial overshadowing. Although unlikely, this possibility could merit a future experiment on context overshadowing that would test after a single conditioning trial. However, the general pattern of results obtained from the present experiments and one in Kwok (2010) suggests that there is a high probability of obtaining a null result from an experiment designed to detect one-trial overshadowing from an event preceding the CS by 10 min or more.

\section{Experiment 3: Comparing overshadowing by pre-CS and post-CS tastes}

Both of the two previous experiments detected overshadowing only after two conditioning sessions. This contrasts with several demonstrations in the taste aversion learning literature of one-trial overshadowing by a second taste. These date back to Revusky's (1971) first demonstration of an interference effect and include Kaye, Gambini, and Mackintosh (1988, Experiment 1) and the study by Bond (1983) discussed above. In a single conditioning session, Bond gave his rats vinegar, followed 10 min later by saline, and then a lithium injection $2 \mathrm{~h}$ later. He found overshadowing of the vinegar aversion by saline, but no overshadowing of the saline aversion by vinegar.

One-trial overshadowing cannot be explained in terms of the cue competition mechanism proposed by Rescorla and Wagner (1972). Consequently, it seems likely that proactive and retroactive interference in taste aversion learning may be based on different underlying processes, as, for example, has been proposed for overshadowing in fear conditioning (Mackintosh \& Reese, 1979). Consequently, the aim of Experiment 3 was to test whether overshadowing by a post-CS taste would be detectable after a single conditioning trial under the conditions in which overshadowing by a preCS taste could be detected only after a second conditioning trial, as in Experiment 1.

The general procedure was similar to that used in Experiment 1, but with no variation in the interval before lithium injection; thus, for all rats, access to sucrose was followed $30 \mathrm{~min}$ later by injection. There were three groups in the single-factor design: Rats received saline before they were given sucrose (pre-CS group), were given saline following sucrose (post-CS group), or were in the control group that was given water instead of saline. As described below, the control group was split into halves that differed as to when they received water, but this did not have a detectable effect.

Method

Subjects Thirty male albino Wistars with no previous experimental history were obtained from the University of Adelaide. They were approximately 72 days old at the start of the experiment, when they weighed a mean of $302 \mathrm{~g}$ (range, 224-370 g). Housing, food, gradual water deprivation, and subsequent access to water, as well as other general procedures, were the same as those in Experiment 1.

Apparatus and solutions The apparatus was the same as in Experiment 1, except that ten transparent acrylic cages were used instead of eight. Drinking solutions were $8 \%$ sucrose and $0.5 \%$ saline, both in tap water.

Procedure Following water pretraining in the drinking chambers (days 1-3), rats were allocated to the three groups (each $n=10$ ), matching for water intakes on day 3. From then on, all sessions lasted $10 \mathrm{~min}$, unless otherwise specified below. On day 4, rats in the control group were given access to $5 \mathrm{ml}$ of saline solution in a 10-min session in the drinking chambers, while those in the pre-CS and post-CS groups were given 10-min access to $5 \mathrm{ml}$ of water.

Conditioning took place on days 5 and 8 . Following the procedure in Experiment 1 for rats in the saline condition, on each of the conditioning days, rats in the pre-CS group were given saline in the drinking chambers for $5 \mathrm{~min}$, returned to their home cages for $10 \mathrm{~min}$, and then placed back in the drinking chambers, where they had access to sucrose for $5 \mathrm{~min}$. The procedure for 5 rats in the control group was exactly the same, except that water was given instead of saline. Rats in the post-CS group were first given sucrose for $5 \mathrm{~min}$, returned to their home cages for $10 \mathrm{~min}$, and then placed back in the drinking chambers to consume saline for $5 \mathrm{~min}$; the remaining control rats were treated in the same way, except that they were given water instead of saline.

All water, sucrose, and saline amounts were limited to $5 \mathrm{ml}$ in the first conditioning session (day 5) and to $15 \mathrm{ml}$ in the second conditioning session (day 8). In both sessions, 30 min after sucrose consumption, rats were given an ip injection of $0.15 \mathrm{M} \mathrm{LiCl}$ at $8 \mathrm{ml} / \mathrm{kg}$ of their body weight. This dose was intermediate between the two used in Experiment 1 and was designed to produce a similar degree of aversion with the intermediate (30-min) delay interval used here. On days $6,7,9$, and 10 , all rats received $10 \mathrm{~min}$ 
of water in the drinking chambers, exactly as during pretraining. At test (day 11), all rats were given sucrose.

\section{Results and discussion}

Water intakes on day 3 (prior to the first sucrose conditioning session), on day 7 (immediately prior to the second conditioning session), and on day 10 (immediately prior to the first sucrose test) were analyzed to test the possibility that the groups might differ in terms of a possible aversion to the neutral context. On the final pretraining day (day 3), the mean (and $S E M$ ) water intakes were the following: preCS, $9.7(0.5) \mathrm{ml}$; post-CS, $9.4(0.7) \mathrm{ml}$; and controls, 9.6 $(0.4), \mathrm{ml}$. A mixed $3 \times 3$ ANOVA, with group and session as factors, applied to these intakes detected no main effects or interactions, largest $F=1.12$, indicating no significant differences between the groups in water intakes from before to after the conditioning treatment.

Analyses of water intakes by the two control subgroups showed that they did not differ on day 3,7 , or 10 , nor did they differ in terms of sucrose intakes on either day 8 or day 11. Independent-sample $t$-tests were conducted to compare water intakes. There were no significant differences in water intakes for the two subgroups on day 3,7 , or 10 , all $t \mathrm{~s}<1$. When $t$-tests were applied to sucrose intakes, there were no significant difference between the subgroups on either day 8 or day 11 , both $t \mathrm{~s}<1$. Since these preliminary analyses failed to reveal any difference in fluid consumption between the two subgroups, their data were combined in subsequent analyses.

The important data are the sucrose intakes shown in Fig. 3. As this figure suggests, the groups did not differ on the first conditioning day, $F<1$. However, on the second conditioning day, the post-CS group drank more sucrose than did the other two groups. An a priori contrast between

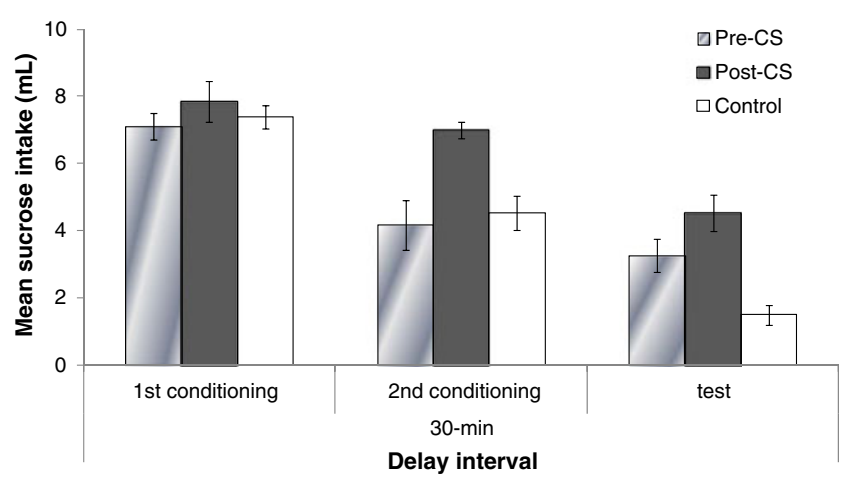

Fig. 3 Experiment 3: Sucrose intakes during the two conditioning sessions and the test session. Rats in the pre-CS group were given saline prior to sucrose, while rats in the post-CS group were given saline after sucrose. Rats in the control group were given water at this time. Lithium injections were given $30 \mathrm{~min}$ following access to sucrose the post-CS and control groups confirmed that sucrose consumption was significantly greater in the post-CS group, $F$ $(1,27)=12.13, p<.025$ (using the Bonferroni adjustment). A similar contrast between the pre-CS and control groups failed to find any difference between them, $F<1$. Thus, as was predicted, one-trial overshadowing was found only in the post-CS group.

Although the pre-CS and control groups did not differ on the second conditioning day, they did differ on the test session (day 11) when both the pre-CS and post-CS groups drank more sucrose than did the controls. When a one-way ANOVA was applied to these data, a large group effect was found, $F(2,27)=11.09$. Analysis of simple effects revealed that the pre-CS and post-CS groups drank more than did the controls, $F(1,27)=7.48$ and $F(1,27)=21.97$, respectively. No significant difference was found between the pre-CS and post-CS groups in the test session, $p=.06$.

In summary, these results confirmed that overshadowing by a subsequent taste (post-CS group) could be detected after a single conditioning trial, while two conditioning trials were needed to reveal overshadowing by the pre-CS taste. This difference is consistent with the proposal that proactive interference with taste aversion learning is based on an associative process (Rescorla \& Wagner, 1972), while retroactive interference is based on some process by which the associability of the target taste is reduced by the occurrence of a subsequent taste. These ideas are explored further in the General Discussion section that follows.

\section{General discussion}

The main aim of this study was achieved in that proactive interference with acquisition of a sucrose aversion was demonstrated both when the interfering stimulus was a taste (Experiment 1) and when it was a novel context (Experiment 2). In Experiments 1 and 3, acquisition of an aversion to sucrose was reduced by prior consumption of saline. Unlike the results of Bond (1983), this overshadowing effect was observed even though a 10-min delay separated consumption of saline and sucrose, reducing the opportunity for direct sensory interactions between the two tastes. In Experiment 2, a similar overshadowing effect was observed when sucrose was preceded by a distinctive context. Taken together, these results clearly indicate that in taste aversion learning, a stimulus presented prior to the target CS still supports overshadowing and, moreover, the effect is not readily attributable to direct perceptual interference, as might be the case when two tastes are presented simultaneously or in immediate succession.

One important difference between this study and Bond (1983) was the number of conditioning trials. The present experiments used two conditioning trials, and in each case, 
proactive overshadowing was not evident in the patterns of consumption after the first trial. Indeed, when proactive and retroactive interference effects were compared in Experiment 3 , one-trial overshadowing was evident only in the retroactive condition. One could conclude from this and past research that one-trial overshadowing is much more likely to occur for the second of two serially presented stimuli. This is the main point on which our results differ from those expected from Revusky's (1971) concurrent interference theory, which predicts interference effects after one trial regardless of whether that interference is proactive or retroactive.

In contrast, the absence of one-trial overshadowing in the proactive conditions is consistent with the analysis of overshadowing provided by Rescorla and Wagner (1972). However, the one-trial overshadowing effect observed in the retroactive condition of Experiment 3 would conventionally be viewed as being inconsistent with the predictions of the model. Nevertheless, there are several ways in which the present results could be accommodated using minimal additional assumptions. One such way is to simply assume different stimulus decay rates depending on what follows after the stimulus, consistent to some extent with Revusky's (1971) notion of interference. To illustrate this more definitively, we developed a simple model of taste aversion learning based on the Rescorla-Wagner model. To account for the present data, the model needs to provide an explanation not only for the difference between proactive and retroactive interference effects after one conditioning trial, but also for the fact that, in Experiment 2, the context supported overshadowing only when there was a short delay of $15 \mathrm{~min}$ between target CS and US. In contrast, in Experiment 1, overshadowing by a taste was still robust with a $90 \mathrm{~min}$ delay. The difference between these results suggests, at the very least, that the influence of the context is relatively short-lived. Thus, trace decay was also assumed to be influenced by the modality of the stimulus. The key assumptions of this model are briefly formalized below.

Trace decay

The associability of the stimulus at the time of conditioning is modulated by a trace decay function. Our use of the word decay refers only to the fact that the stimulus trace is diminishing, whether it be as a consequence of time passing, the presence of other interfering stimuli, or both. Thus, our definition departs from the conventional use of the term to denote the change caused purely by the passage of time. We chose an exponential decay function because it naturally follows from the formulae of many real-time models of associative learning (e.g., Harris \& Livesey, 2010; McLaren \& Mackintosh, 2000). However, in principle, similar results could be achieved with either linear or sigmoidal functions.
The surviving stimulus trace, $\gamma_{\mathrm{CS}}$, at a given time after stimulus offset is described by

$\gamma_{C S}=e^{-k t}$,

where $t$ is the time elapsed (in this case, in hours) since the removal of the stimulus, and $k$ is a constant that we specifically vary for contextual versus taste stimuli to alter the rate of decay. Hence, $\gamma_{\mathrm{CS}}$ varies between 0 and 1 and affects the associability of the stimulus accordingly. We made two simple assumptions about rates of decay of the different stimuli: (1) Stimuli decay at a faster rate if they are followed by the presentation of other stimuli, and (2) stimuli in different modalities decay at different rates - specifically, the context conditioned in Experiment 2 decays faster than the sucrose and saline flavors. Varying decay rates are implemented by changing the value of $k$, as is shown in Fig. 4. We chose $k=0.1$ for a flavor that is not followed by other stimuli (unmasked flavor), $k=0.25$ for the first of two serially presented flavors (i.e., a flavor that is masked by a second stimulus), and $k=0.6$ for the context used in Experiment 2. In Fig. 4, the function in Eq. 1 is shown for each of these three values of $k$, and with dotted vertical lines indicating several critical time points used in the simulations for Experiments 1 and 2. Where taste and/or contexts were experienced sequentially, we assumed that the offset of the

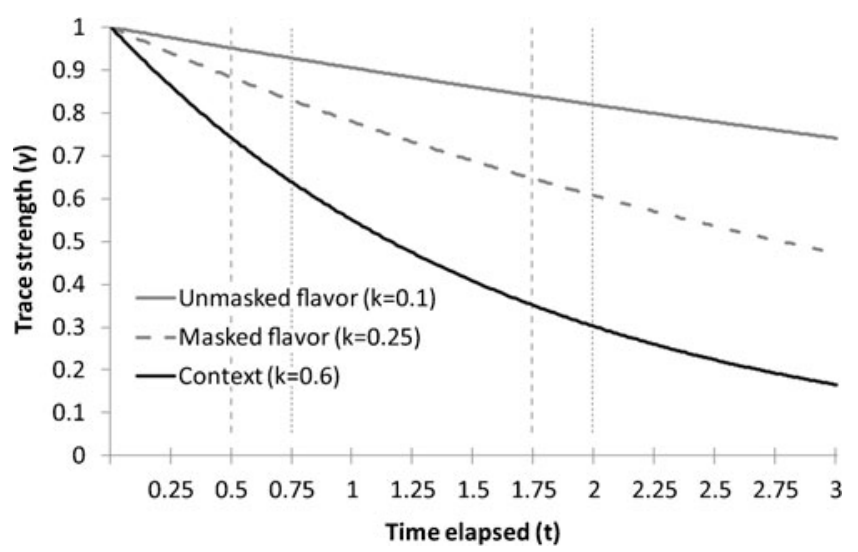

Fig. 4 Stimulus trace strength $(\gamma)$ as a function of time elapsed in hours since the removal of the stimulus $(t)$. Lines plot the function $\gamma=e^{-k t}$ for the three values of the constant $k$ used in the simulations of Experiments $1-3$. The dashed and dotted vertical lines provide examples of $t$ values used in the 15- and 90-min conditions. Dashed vertical lines mark the time points at which the unconditioned stimulus (US) was assumed to take effect relative to offset of sucrose consumption ( $t=0.5$ and $t=1.75$, yielding $\gamma_{\mathrm{suc}}=.95$ and .84 , where sucrose is an unmasked flavor). Dotted lines mark the time points at which the US was assumed to take effect relative to offset of either saline consumption or context $(t=0.75$ and $t=$ 2.0 , yielding $\gamma_{\text {sal }}=.83$ and $.61 ; \gamma_{\text {context }}=.64$ and .30$)$. Note that saline in Experiment 1 was considered a masked flavor $(k=0.25)$ since it was followed by sucrose consumption, whereas in Experiment 3, the first of two solutions (sal in proactive condition and suc in retroactive condition) was considered masked $(k=0.25)$ and the second/only taste was considered unmasked $(k=0.1)$ 
first stimulus preceded offset of the second by $15 \mathrm{~min}$ $(0.25 \mathrm{~h})$. Furthermore, in each instance of conditioning, we assumed that a further 15 min elapsed between the lithium injection and the onset of the effective US (nausea). For instance, in the 30-min saline condition in Experiment 1, we assumed that the rats ceased drinking sucrose $45 \mathrm{~min}(t=$ $0.75)$ before the onset of nausea and ceased drinking saline $60 \min (t=1)$ before the onset of nausea.

\section{Learning}

The associative strength of each CS $\left(V_{\mathrm{CS}}\right)$ was updated in a trial-by-trial fashion, just as in the Rescorla-Wagner model. The learning rule itself was slightly modified to incorporate the strength of the trace. Thus, changes in associative strength $(\Delta V)$ were calculated according to Eq. 2:

$\Delta V_{c S}=\gamma_{C S} \times \alpha_{C S} \times \beta_{U S} \times(\lambda-\Sigma V)$.

Here, $\alpha$ and $\beta$ are learning rate parameters reflecting the intrinsic associability of the CS and US, respectively, while $\lambda$ reflects the level of reinforcement supported by the US. We used $\alpha=1, \beta=0.5$, and $\lambda=1$ in all simulations in order to simplify the model as much as possible. Thus, learning was influenced only by the strength of the CS trace $\left(\gamma_{\mathrm{CS}}\right)$ and the error term $(\lambda-\Sigma V)$, the difference between $\lambda$ and the extent to which this reinforcement was anticipated $(\Sigma V)$. The calculation of $\Sigma V$ was slightly modified to account for decay of the CS traces that elicit the expectation of the US. Thus, for $n$ stimuli with active traces,

$\Sigma V=\sum_{i=1}^{n}\left(\gamma_{i} \times V_{i}\right)$

Figure 5 shows a simulation of test data for each of the three experiments plotted against the actual empirical data. Each plot of simulated data shows $e^{-}{ }^{V}$, which gives a value of 1 when $V=0$ and approaches zero as $V$ increases, thus giving an indication of predicted consumption. In these simulations, only the value of $k$ was varied. The values of $k$ were selected fairly arbitrarily to reflect (1) the hypothesis that taste stimuli decay more slowly than others, and (2) the assumption that stimuli interfere with the traces of those that precede them. The value of $k$ in the decay function for a context affects the simulated results of Experiment 2. Here,
Fig. 5 Empirical and simulated test data for each of the three experiments. Left panels show intake of sucrose on test from each experiment. Right panels show predicted intake from simulations. Simulated data show $e^{-V}$, where $V$ equals the associative strength of the sucrose after conditioning. For Experiments 1 and 2, the data represent sucrose intakes in the test session following two conditioning sessions. For Experiment 3, intakes are shown for both the second conditioning session and the test
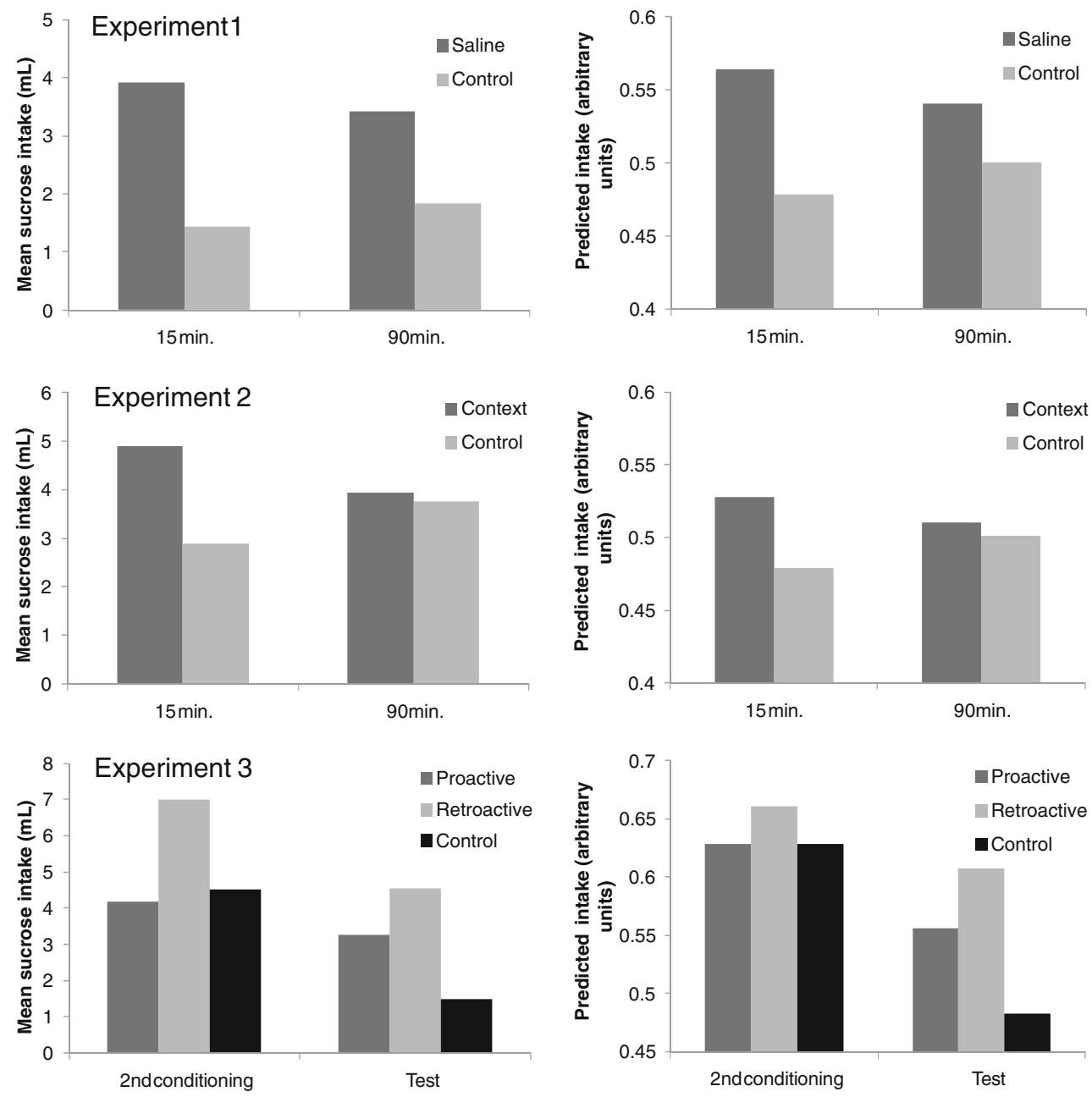
one may have reason to assume that the context should have a lower salience than each of the flavors. Thus, it is worth noting that a very similar result is obtained if $\alpha$ for the context is less than $\alpha$ for the sucrose flavor. The value of $k$ in the decay function for a masked flavor (i.e., the first of the two serially presented flavors) affects the simulated results of Experiments 1 and 3.

It is noteworthy that in Experiment 3, the predicted difference between the retroactive and control conditions after one conditioning trial is not generated by the same mechanism as the predicted overshadowing effects evident after two conditioning trials in all three experiments. Instead, it is simply the result of a weaker sucrose trace, which is predicted to decay faster in the retroactive condition due to the subsequent presentation of saline. This difference is more pronounced if the masked flavor trace is made to decay more quickly (higher values of $k$ ). However, doing so also adversely weakens the overshadowing effect observed in the 90-min condition of Experiment 1 (slower decay of the masked flavor leads to stronger overshadowing). Therefore, $k=0.25$ represents a compromise between the results of these two experiments. Nevertheless, the general pattern of results found in the empirical data is matched relatively well by the simulations.

Although the analysis above provides a very good account of the present results concerning proactive interference, it also assumes that the evidence for one-trial overshadowing in taste aversion learning is due to direct interference with the trace of the target stimulus, and not because of the cue competition mechanism implemented in the Rescorla-Wagner model. Several studies have found one-trial serial overshadowing of the first of two flavors. For instance, in the first demonstration of his theory of concurrent interference, Revusky (1971) arranged that saccharin would be followed 15 min later by either water or different concentrations of vinegar; $60 \mathrm{~min}$ after this second drink, the rats were injected with a strong dose of lithium. The results from three saccharin tests following the initial conditioning session indicated that the vinegar had interfered with acquisition of the saccharin aversion to an extent that increased with vinegar concentration. A similar one-trial overshadowing effect was reported by Kaye et al. (1988, Experiment 1) for retroactive interference by sucrose with 4-h delay conditioning of a vinegar aversion. Furthermore, a one-trial retroactive interference effect has also been reported in a shock-based trace conditioning study in which a 15 -s tone was followed by a shock $30 \mathrm{~s}$ later; a 15 -s light immediately preceding the shock was found to interfere with one-trial conditioning of fear of the tone (Mackintosh \& Reese, 1979, Experiment 2). These authors suggested that overshadowing can arise from at least two different processes. The first is that described by Rescorla and Wagner (1972), which, as was detailed above, accounts for overshadowing across multiple conditioning trials, while the second reflects competition between stimuli for attention and explains overshadowing that occurs in a single conditioning trial. Thus, according to their account, in the fear-conditioning experiment we have just described, the occurrence of the light would have the effect of decreasing the associability of the preceding tone, and in Revusky's retroactive interference experiment, the vinegar would have decreased the associability of the preceding saccharin. This explanation has obvious parallels with the account that we have just provided, although we have made use of quantitative changes in trace strength without necessarily assuming that these changes are related to attention per se. In the particular case of taste aversion learning, it is quite possible that trace decay represents very gradual perceptual and memorial depletion over time and that both may be subject to retroactive interference. But in any case, a twoprocess account of overshadowing could explain why onetrial retroactive interference effects can be found, whereas no one-trial proactive interference was found in the present experiments.

We have chosen to focus on the Rescorla-Wagner model in this report because it is perhaps the most widely understood model of Pavlovian conditioning that deals explicitly with overshadowing phenomena. Its simplicity allows us to clearly illustrate the potential impact of a single additional factor (differential trace decay) on a widely accepted psychological mechanism (learning based on prediction error). It is not our intention to rule out other potential explanations for these effects. For example, instead of the acquisitiondeficit account of overshadowing that is assumed here, it is possible that one based on retrieval deficits, such as a comparator theory (e.g., Denniston, Savastano, \& Miller, 2001), could be modified to fit the present results. Furthermore, several real-time models based on learning mechanisms similar to the Rescorla-Wagner model can account for these data via processes similar to those we have outlined here (e.g., Harris \& Livesey, 2010; McLaren \& Mackintosh, 2000; Wagner, 1981). By their very nature, these models provide scope for quantitative differences in trace decay, although they make different assumptions about how stimulus representations might interfere with one another. Unlike the trial-based formulation of the Rescorla-Wagner model, realtime models do tend to predict at least some one-trial overshadowing due to the manner in which a learning episode is broken into small time steps or microtrials, updating prediction error incrementally. However, this predicted one-trial effect is typically weaker relative to multiple-trial learning, especially when trace conditioning is simulated using models that explicitly assume some form of stimulus interference (e.g., Harris \& Livesey, 2010). The clearest reason why a real-time error correction model would predict more onetrial overshadowing for the first of two serially presented stimuli than for the second is that the representation of the second stimulus disrupts the representation of the first. This is 
consistent with the assumptions we have used in the present model.

In addition to their contribution toward understanding long-delay learning, the present results also have implications for real-world situations - most noticeably, in cancer chemotherapy. Despite advances in pharmacological control of chemotherapy-induced sickness, up to $80 \%$ of patients experience some form of nausea (Roscoe, Morrow, Hickok, $\&$ Stern, 2000). Both nausea and vomiting induced by chemotherapy can be anticipatory (ANV; Hall \& Symonds, 2006) and are linked to the development of psychological side effects, developed through associative processes similar to that of Pavlovian conditioning and resembling conditioned taste aversion mechanisms (Bernstein, 1978). Both simple and effective ways to reduce the impact of chemotherapy are needed, given that the failure to control ANV may induce delays between cycles of treatments, reduce the effectiveness of treatment, and increase the likelihood that patients will drop out (Miller \& Kearney, 2004).

The results from the present study support the use of interference as a theoretical model for the control of ANV. Following Bernstein (1978), Stockhorst, Wiener, Klosterhalfen, Klosterhalfen, and Steingruber (1998) used an overshadowing protocol with cancer patients undergoing chemotherapy with the aim of preventing - or at least reducing-ANV. In their study, a novel stimulus, a flavored drink, was introduced as a "scapegoat" to prevent the stimuli typically occurring during treatment (tastes, smells, and contexts) from becoming nausea-eliciting stimuli. The authors found that none of the patients in the overshadowing condition developed ANV, whereas $23 \%$ of the control group did so. However, a problem with this approach was that many patients refused to drink the novel solution, explaining that they were already feeling nauseous by the time the solution was offered. This could be avoided if the solution was given before treatment, when, according to the present results, it would still produce interference with ANV. Although the solution would itself become unpleasant to consume, it could be very effective in mitigating the more debilitating consequences of ANV to other cues associated with treatment.

In conclusion, the most important outcomes from this study were to provide the first analysis of the interference effects identified by Revusky (1971) that occur in long-delay learning and to provide the first quantitative model of long-delay learning by incorporating decay functions into the Rescorla-Wagner (1972) model of associative learning.

Author notes This research was partly supported by an Australian Research Council grant to R.A.B. The authors are grateful to Justin Harris, Nick Mackintosh, and Fred Westbrook for their comments on an earlier version of the manuscript.

\section{References}

Baddeley, A. D. (1990). Human memory: Theory and practice. Hove, U.K.: Erlbaum.

Bernstein, I. L. (1978). Learned taste aversions in children receiving chemotherapy. Science, 200, 1302-1303.

Bond, N. W. (1983). Reciprocal overshadowing in flavour-aversion learning. Quarterly Journal of Experimental Psychology, 35, 265-274.

Denniston, J. C., Savastano, H. I., \& Miller, R. R. (2001). The extended comparator hypothesis: Learning by contiguity, responding by relative strength. In R. R. Mowrer \& S. B. Klein (Eds.), Handbook of contemporary learning theories (pp. 65-117). Mahwah, NJ: Erlbaum.

Freeman, K. B., \& Riley, A. L. (2009). The origins of conditioned taste aversion: A historical analysis. In S. Reilly \& T. D. Schachtman (Eds.), Conditioned taste aversion: Behavioral and neural processes (pp. 9-33). New York: Oxford University Press.

Garcia, J., Kimeldorf, D. J., \& Koelling, R. A. (1955). Conditioned aversion to saccharin resulting from exposure to gamma radiation. Science, 122, 318-321.

Garcia, J., \& Koelling, R. A. (1966). Relation of cue to consequence in avoidance learning. Psychonomic Science, 4, 123-124.

Gleitman, H., \& Jung, L. (1963). Retention in rats: The effect of proactive interference. Science, 142, 1683-1684.

Grice, G. R. (1948). The relation of secondary reinforcement to delayed reward in visual discrimination learning. Journal of Experimental Psychology, 38, 1-16.

Hall, G., \& Symonds, M. (2006). Overshadowing and latent inhibition of context aversion conditioning in the rat. Autonomic Neuroscience, $129,42-49$.

Harris, J. A., \& Livesey, E. J. (2010). An attention-modulated associative network. Learning \& Behavior, 38, 1-26.

Kaye, H., Gambini, B., \& Mackintosh, N. J. (1988). A dissociation between one-trial overshadowing and the effect of a distractor on habituation. Quarterly Journal of Experimental Psychology, 40B, 31-47.

Kimble, G. A. (1961). Hilgard and Marquis' conditioning and learning. New York: Appleton-Century-Crofts.

Kwok, D. W. S. (2010). Taste aversion learning and interference in the rat. Unpublished honours thesis submitted to the School of Psychology, University of Sydney.

Kwok, D. W. S., \& Boakes, R. A. (in press). Blocking of acquisition of a taste aversion by a context experienced prior to the taste. Behavioural Processes.

Loftus, E. F. (1980). Memory. Reading, MA: Addison-Wesley. (Reprinted 1988 by ArdsleyPress, New York)

Mackintosh, N. J. (1974). The psychology of animal learning. London: Academic Press.

Mackintosh, N. J., \& Reese, B. (1979). One-trial overshadowing. Quarterly Journal of Experimental Psychology, 31, 519526.

McGeoch, J. A., \& Irion, A. L. (1952). The psychology of human learning. New York: Longmans, Green.

McLaren, I. P. L., \& Mackintosh, N. J. (2000). Associative learning and elemental representations: I. A theory and its application to latent inhibition and perceptual learning. Animal Learning \& Behavior, 26, 211-246.

Miller, M., \& Kearney, N. (2004). Chemotherapy-related nausea and vomiting - Past reflections, present practice and future management. European Journal of Cancer Care, 13, 71-78.

Pavlov, I. P. (1927). Conditioned reflexes. London: Oxford University Press.

Postman, L. (1975). Verbal learning and memory. Annual Review of Psychology, 26, 291-335. 
Rescorla, R. A., \& Wagner, A. R. (1972). A theory of Pavlovian conditioning: Variations in the effectiveness of reinforcement and nonreinforcement. In A. H. Black \& W. F. Prokasy (Eds.), Classical conditioning II: Current research and theories (pp. 6499). New York: Appleton Century-Crofts.

Revusky, S. (1971). The role of interference in association over a delay. In W. K. Honig \& P. H. R. James (Eds.), Animal memory (pp. 155-213). New York: Academic Press.

Roscoe, J. A., Morrow, G. R., Hickok, J. T., \& Stern, R. M. (2000). Nausea and vomiting remain a significant problem: Trends over time in controlling chemotherapy-induced nausea and vomiting in 1413 patients treated in community clinical practices. Journal of Pain and Symptom Management, 20, 113-121.
Shanks, D. R., Pearson, S. M., \& Dickinson, A. (1989). Temporal contiguity and the judgment of causality. Quarterly Journal of Experimental Psychology, 41B, 139-159.

Stockhorst, U., Wiener, J. A., Klosterhalfen, S., Klosterhalfen, W., Aul, C., \& Steingruber, H. J. (1998). Effects of overshadowing on conditioned nausea in cancer patients. Physiology \& Behavior, 64, 743-753.

Underwood, B. J. (1957). Interference and forgetting. Psychological Review, 64, 49-60.

Wagner, A. R. (1981). SOP: A model of automatic memory processing in animal behavior. In N. E. Spear \& R. R. Miller (Eds.), Informal processing in animals: Memory mechanisms (pp. 5-47). Hillsdale, NJ: Erlbaum. 